\title{
Cosmological constant and vacuum energy
}

\author{
G. E. Volovik* \\ Helsinki University of Technology, Low Temperature Laboratory, \\ P.O. Box 2200, FIN-02015 HUT, Finland, \\ Landau Institute for Theoretical Physics, 119334 Moscow, Russia
}

(October 18, 2004)

\begin{abstract}
The general thermodynamic analysis of the quantum vacuum, which is based on our knowledge of the vacua in condensed-matter systems, is consistent with the Einstein earlier view on the cosmological constant. In the equilibrium Universes the value of the cosmological constant is regulated by matter. In the empty Universe, the vacuum energy is exactly zero, $\lambda=0$. The huge contribution of the zero point motion of the quantum fields to the vacuum energy is exactly cancelled by the higher-energy degrees of freedom of the quantum vacuum. In the equilibrium Universes homogeneously filled by matter, the vacuum is disturbed, and the energy density of the vacuum becomes proportional to that of matter, $\lambda=\rho_{\mathrm{vac}} \sim \rho_{\text {matter }}$. This consideration applies to any vacuum in equilibrium irrespective of whether the vacuum is false or true, and is valid both in Einstein's general theory of relativity and within the special theory of relativity, i.e. in a world without gravity.
\end{abstract}

\footnotetext{
*Email address: volovik@boojum.hut.fi
} 


\section{INTRODUCTION}

In 1917, Einstein proposed a model of our Universe [1]. To make the Universe static, he introduced the famous cosmological constant which was counterbalancing the collapsing tendency of the gravitating matter. As a static solution of the field equations of general relativity with added cosmological term, he obtained the Universe with spatial geometry of a three-dimensional sphere. In Einstein treatment the cosmological constant is universal, i.e. it must be constant throughout the whole Universe. But it is not fundamental: its value is determined by the matter density in the Universe. In Ref. [2], Einstein noted that the $\lambda$-term must be added to his equations if the density of matter in the Universe is non-zero in average. In particular, this means that $\lambda=0$ if matter in the Universe is so inhomogeneously distributed that its average over big volumes $V$ tends to zero. In this treatment, $\lambda$ resembles a Lagrange multiplier or an integration constant, rather than the fundamental constant (see general discussion in Ref. [3,4]).

With the development of the quantum field theory it was recognized that the $\lambda$-term is related to zero-point motion of quantum fields. It describes the energy-momentum tensor of the quantum vacuum, $T_{\text {vac }}^{\mu \nu}=\lambda g^{\mu \nu}$. This means that $\lambda$ is nothing but the energy density of the vacuum, $\lambda=\rho_{\mathrm{vac}}$, i.e. the vacuum can be considered as a medium obeying the equation of state:

$$
\rho_{\mathrm{vac}}=-p_{\mathrm{vac}}
$$

Such view on the cosmological constant led to principle difficulties. The main two problems are: (i) the energy density of the zero-point motion is highly divergent because of the formally infinite number of modes; (ii) the vacuum energy is determined by the high-energy degrees

of quantum fields, and thus at first glance must have a fixed value which is not sensitive to the low-density and low-energy matter in the present Universe, which is also in disagreement with observations.

The naive summation over all the known modes of the quantum fields gives the following estimate for the energy density of the quantum vacuum 


$$
\rho_{\mathrm{vac}}=\frac{1}{V}\left(\frac{1}{2} \sum_{b} \sum_{\mathbf{p}} E_{b}(p)-\sum_{f} \sum_{\mathbf{p}} E_{f}(p)\right) .
$$

Here the negative contribution comes from the negative energy levels occupied by fermionic species $f$ in the Dirac sea; the positive contribution comes from the zero-point energy of quantum fluctuations of bosonic fields $b$. Since the largest contribution comes from the quantum fluctuations with ultrarelativistic momenta $p \gg m c$, the masses $m$ of particles can be neglected, and the energy spectrum of particles can be considered as massless, $E_{b}(p)=$ $E_{f}(p)=c p$. Then the energy density of the quantum vacuum is expressed in terms of the number $\nu_{b}$ and $\nu_{f}$ of bosonic and fermionic species:

$$
\rho_{\mathrm{vac}}=\frac{1}{V}\left(\frac{1}{2} \nu_{b} \sum_{\mathbf{p}} c p-\nu_{f} \sum_{\mathbf{p}} c p\right) \sim \frac{1}{c^{3}}\left(\frac{1}{2} \nu_{b}-\nu_{f}\right) E_{\mathrm{Pl}}^{4} .
$$

Here $E_{\mathrm{Pl}}$ is the Planck energy cut-off. This estimate of the cosmological constant exceeds by 120 orders of magnitude the upper limit posed by astronomical observations. The more elaborated calculations of the vacuum energy, which take into account the interaction between different modes in the vacuum, can somewhat reduce the estimate but not by many orders of magnitude. The supersymmetry - the symmetry between the fermions and bosons which imposes the relation $\nu_{b}=2 \nu_{f}$ - does not help too. In our world the supersymmetry is not exact, and one obtains $\rho_{\mathrm{vac}} \sim \frac{1}{c^{3}} E_{\mathrm{UV}}^{4}$, where the ultra-violet cut-off $E_{\mathrm{UV}}$ is provided by the energy scale below which the supersymmetry is violated. If it exists, the supersymmetry can substantially reduce this estimate, but still a discrepancy remains of at least 60 orders of magnitude.

Moreover, the cut-off energy is the intrinsic parameter of quantum field theory. It is determined by the high energy degrees of freedom of the order of $E_{\mathrm{UV}}$ or $E_{\mathrm{Pl}}$ and thus cannot be sensitive to the density of matter in the present Universe. The typical energies of the present matter are too low compared to $E_{\mathrm{UV}}$, and thus the matter is unable to influence such a deep structure of the vacuum. This contradicts to recent observations which actually support the Einstein prediction that the cosmological constant is determined by the energy density of matter $\rho_{\text {matter. }}$. At the moment the consensus has emerged about the experimental 
value of the cosmological constant $[5,4]$. It is on the order of magnitude of the matter density, $\rho_{\text {vac }} \sim 2-3 \rho_{\text {matter. }}$. This is comparable to

$$
\rho_{\mathrm{vac}}=\frac{1}{2} \rho_{\text {matter }}
$$

obtained by Einstein for the static cold Universe, and

$$
\rho_{\mathrm{vac}}=\rho_{\text {matter }}
$$

in the static hot Universe filled by ultra-relativistic matter or radiation (see Eq. (7.5) below).

The pressure of the vacuum was found to be negative, $p_{\mathrm{vac}}=-\rho_{\mathrm{vac}}<0$, which means that the vacuum does really oppose and partially counterbalance the collapsing tendency of matter. This demonstrates that, though our Universe is expanding (even with acceleration) and is spatially flat, it is not very far from the Einstein's static equilibrium solution.

The problem is how to reconcile the astronomical observations with the estimate of the vacuum energy imposed by the relativistic Quantum Field Theory (QFT). What is the flaw in the arguments which led us to Eq. (1.3) for the vacuum energy? The evident weak point is that the summation over the modes in the quantum vacuum is constrained by the cut-off: we are not able to sum over all degrees of freedom of the quantum vacuum since we do not know the physics of the deep vacuum beyond the cut-off. It is quite possible that we simply are not aware of some very simple principles of the trans-Planckian physics from which it immediately follows that the correct summation over all the modes of the quantum vacuum gives zero or almost zero value for the vacuum energy density, i.e. the trans-Planckian degrees of freedom effectively cancel the contribution of the sub-Planckian degrees irrespective of details of trans- and sub-Planckian physics. People find it easier to believe that such an unknown mechanism of cancellation if it existed would reduce $\lambda$ to exactly zero rather than the observed very low value.

Since we are looking for the general principles governing the energy of the vacuum, it should not be of importance for us whether the QFT is fundamental or emergent. Moreover, we expect that these principles should not depend on whether or not the QFT obeys all 
the symmetries of the relativistic QFT: these symmetries (Lorentz and gauge invariance, supersymmetry, etc.) still did not help us to nullify the vacuum energy). That is why to find these principles we can look at the quantum vacua whose microscopic structure is well known at least in principle. These are the ground states of the quantum condensed-matter systems, such as superfluid liquids, Bose-Einstein condensates in ultra-cold gases, superconductors, insulators, systems experiencing the quantum Hall effect, etc. These systems provide us with a broad class of Quantum Field Theories which are not restricted by Lorentz invariance. This allows us to consider many problems in the relativistic Quantum Field Theory of the weak, strong and electromagnetic interactions and gravitation from a more general perspective. In particular, the cosmological constant problems: Why is $\lambda$ not big? Why is it non-zero? Why is it of the order of magnitude of the matter density? ...

\section{EFFECTIVE QFT IN QUANTUM LIQUIDS}

The homogeneous ground state of a quantum system, even though it contains a large amount of particles (atoms or electrons), does really play the role of a quantum vacuum. Quasiparticles - the propagating low-frequency excitations above the ground state, that play the role of elementary particles in the effective QFT - see the ground state as an empty space. For example, phonons - the quanta of the sound waves in superfluids - do not scatter on the atoms of the liquid if the atoms are in their ground state. The interacting bosonic and fermionic quasiparticles are described by the bosonic and fermionic quantum fields, obeying the same principles of the QFT except that in general they are not relativistic and do not obey the symmetries of relativistic QFT. They obey at most the Galilean invariance and have a preferred reference frame where the liquid is at rest. It is known, however, that in some of these systems the effective Lorentz symmetry emerges for quasiparticles. Moreover, if the system belongs to a special universality class, the Lorentz symmetry emerges together with effective gauge and metric fields [6]. This fact, though encouraging for other applications of condensed matter methods to relativistic QFT (see e.g. [7]), is not important for our 
consideration. The principle which leads to nullification of the vacuum energy is more general, it comes from a thermodynamic analysis which is not constrained by symmetry or universality class.

To see it let us consider two quantum vacua: the ground states of two quantum liquids, superfluid ${ }^{4} \mathrm{He}$ and one of the two superfluid phases of ${ }^{3} \mathrm{He}$, the A-phase. We have chosen these two liquids because the spectrum of quasiparticles playing the major role at low energy is 'relativistic', i.e. $E(p)=c p$, where $c$ is some parameter of the system. This allows us to make the connection to relativistic QFT. In superfluid ${ }^{4} \mathrm{He}$ the relevant quasiparticles are phonons (quanta of sound waves), and $c$ is the speed of sound. In superfluid ${ }^{3} \mathrm{He}-\mathrm{A}$ the relevant quasiparticles are fermions. The corresponding 'speed of light' $c$ (the slope in the linear spectrum of these fermions) is anisotropic; it depends on the direction of their propagation: $E^{2}(\mathbf{p})=c_{x}^{2} p_{x}^{2}+c_{y}^{2} p_{y}^{2}+c_{z}^{2} p_{z}^{2}$. But this detail is not important for our consideration.

According to the naive estimate in Eq. (1.3) the density of the ground state energy in the bosonic liquid ${ }^{4} \mathrm{He}$ comes from the zero-point motion of the phonons

$$
\rho_{\mathrm{vac}}=\frac{1}{2} \sum_{\mathbf{p}} c p \sim \frac{E_{\mathrm{Pl}}^{4}}{c^{3}}=E_{\mathrm{Pl}}^{4} \sqrt{-g},
$$

where the ultraviolet cut-off is provided by the Debye temperature, $E_{\mathrm{Pl}}=E_{\text {Debye }} \sim 1 \mathrm{~K}$; $c \sim 10^{4} \mathrm{~cm} / \mathrm{s}$; and we introduced the effective acoustic metric for phonons [8]. The ground state energy of fermionic liquid must come from the occupied negative energy levels of the Dirac sea:

$$
\rho_{\mathrm{vac}} \sim-\frac{E_{\mathrm{Pl}}^{4}}{c_{x} c_{y} c_{z}}=-E_{\mathrm{Pl}}^{4} \sqrt{-g} .
$$

Here the 'Planck' cut-off is provided by the amplitude of the superfluid order parameter, $E_{\mathrm{Pl}}=\Delta \sim 1 \mathrm{mK} ; c_{z} \sim 10^{4} \mathrm{~cm} / \mathrm{s} ; c_{x}=c_{y} \sim 10 \mathrm{~cm} / \mathrm{s}$

These estimates were obtained by using the effective QFT for the 'relativistic' fields. Comparing them with the results obtained by using the known microscopic physics of these liquids one finds that these estimates are not completely crazy: they do reflect some important part of microscopic physics. For example, the Eq. (2.2) gives the correct order of 
magnitude for the difference between the energy densities of the liquid ${ }^{3} \mathrm{He}$ in superfluid state, which represents the true vacuum, and the normal (non-superfluid) state representing the false vacuum:

$$
\rho_{\text {true }}-\rho_{\text {false }} \sim-\frac{E_{\mathrm{Pl}}^{4}}{c_{x} c_{y} c_{z}}
$$

However, it says nothing on the total energy density of the liquid. Moreover, as we shall see below, it also gives a disparity of many orders of magnitude between the estimated and measured values of the analog of the cosmological constant in this liquid. Thus in the condensed-matter vacua we have the same paradox with the vacuum energy. But the advantage is that we know the microscopic physics of the quantum vacuum in these systems and thus are able to resolve the paradox there.

\section{RELEVANT THERMODYNAMIC POTENTIAL FOR QUANTUM VACUUM}

When one discusses the energy of condensed matter, one must specify what thermodynamic potential is relevant for the particular problem which he or she considers. Here we are interested in the analog of the QFT emerging in condensed matter. The many-body system of the collection of identical atoms (or electrons) obeying Schrödinger quantum mechanics can be described in terms of the QFT [9] whose Hamiltonian is

$$
\mathcal{H}-\sum_{a} \mu_{a} \mathcal{N}_{a}
$$

Here $\mathcal{H}$ is the second-quantized Hamiltonian of the many-body system containing the fixed numbers of atoms of different sorts. It is expressed in terms of the Fermi and Bose quantum fields $\psi_{a}(r, t)$. The operator $\mathcal{N}_{a}=\int d^{3} \psi_{a}^{\dagger} \psi_{a}$ is the particle number operator for atoms of sort a. The Hamiltonian (3.1) removes the constraint imposed on the quantum fields $\psi_{a}$ by the conservation law for the number of atoms of sort $a$, and it corresponds to the thermodynamic potential with fixed chemical potentials $\mu_{a}$. The Hamiltonian (3.1) also serves as a starting point for the construction of the effective QFT for quasiparticles, and thus it is responsible for their vacuum. 
Thus the correct vacuum energy density for the QFT emerging in the many-body system is determined by the vacuum expectation value of fhe Hamiltonian (3.1) in the thermodynamic limit $V \rightarrow \infty$ and $N_{a} \rightarrow \infty$ :

$$
\rho_{\mathrm{vac}}=\frac{1}{V}\left\langle\mathcal{H}-\sum_{a} \mu_{a} \mathcal{N}_{a}\right\rangle_{\mathrm{vac}} .
$$

One can check that this is the right choice for the vacuum energy using the Gibbs-Duhem relation of thermodynamics. It states that if the condensed matter is in equilibrium it obeys the following relation between the energy $E=\langle\mathcal{H}\rangle$, and the other thermodynamic varaibles - the temperature $T$, the entropy $S$, the particle numbers $N_{a}=\left\langle\mathcal{N}_{a}\right\rangle$, the chemical potentials $\mu_{a}$, and the pressure $p$ :

$$
E-T S-\sum_{a} \mu_{a} N_{a}=-p V
$$

Applying this thermodynamic Gibbs-Duhem relation to the ground state at $T=0$ and using Eq. (3.2) one obtains

$$
\rho_{\mathrm{vac}}=\frac{1}{V}\left(E-\sum_{a} \mu_{a} N_{a}\right)_{\mathrm{vac}}=-p_{\mathrm{vac}} .
$$

Omitting the intermediate expression, the second term in Eq. (3.4) which contains the microscopic parameters $\mu_{a}$, one finds the familiar equation of state for the vacuum - the equation (1.1). The vacuum is a medium with the equation of state (1.1), and such a medium naturally emerges in any condensed-matter QFT, relativistic or non-relativistic. This demonstrates that the problem of the vacuum energy can be considered from the more general perspective not constrained by the relativistic Hamiltonians. Moreover, it is not important whether there is gravity or not. We shall see below in Sec. V that the vacuum plays an important role even in the absence of gravity: it stabilizes the Universe filled with hot non-gravitating matter

There is one lesson from the microscopic consideration of the vacuum energy, which we can learn immediately and which is very important for one of the problems related to the cosmological constant. The problem is that while on the one hand the physical laws 
do not change if we add a constant to the Hamiltonian, i.e. they are invariant under the transformation $\mathcal{H} \rightarrow \mathcal{H}+C$, on the other hand gravity responds to the whole energy and thus is sensitive to the choice of $C$. Our condensed-matter QFT shows how this problem can be resolved. Let us shift the energy of each atom of the many-body system by the same amount $\alpha$. This certainly changes the original many-body Hamiltonian $\mathcal{H}$ for the fixed number of atoms: after the shift it becomes $\mathcal{H}+\alpha \sum_{a} N_{a}$. But the proper Hamiltonian (3.1), which is relevant for the QFT, remains invariant under this transformation, $\mathcal{H}-\sum_{a} \mu_{a} \mathcal{N}_{a} \rightarrow$ $\mathcal{H}-\sum_{a} \mu_{a} \mathcal{N}_{a}$. This is because the chemical potentials are also shifted: $\mu_{a} \rightarrow \mu_{a}+\alpha$.

This demonstrates that when the proper thermodynamic potential is used, the vacuum energy becomes independent of the choice of the reference for the energy. This is the general thermodynamic property which does not depend on details of the many-body system. This suggests that one of the puzzles of the cosmological constant - that $\lambda$ depends on the choice of zero energy level - could simply result from our very limited knowledge of the quantum vacuum. We are unable to see the robustness of the vacuum energy from our low-energy corner, we need a deeper thermodynamic analysis. But the result of this analysis does not depend on the details of the structure of the quantum vacuum. In particular, it does not depend on how many different chemical potentials $\mu_{a}$ are at the microscopic level: one, several or none. That is why we expect that this general thermodynamic analysis could be applied to our vacuum too.

\section{NULLIFICATION OF VACUUM ENERGY IN THE EQUILIBRIUM VACUUM}

Now let us return to our two monoatomic quantum liquids, ${ }^{3} \mathrm{He}$ and ${ }^{4} \mathrm{He}$, each with a single chemical potential $\mu$, and calculate the relevant ground-state energy (3.2) in each of them. Let us consider the simplest situation, when our liquids are completely isolated from the environment. For example, one can consider the quantum liquid in space where it forms a droplet. Let us assume that the radius $R$ of the droplet is so big that we can neglect the 
contribution of the surface effects to the energy density. The evaporation at $T=0$ is absent, that is why the ground state exists and we can calculate its energy from the first principles. Though both liquids are collections of strongly interacting and strongly correlated atoms, numerical simulations of the ground state energy have been done with a very simple result. In the limit $R \rightarrow \infty$ and $T=0$ the energy density of both liquids $\rho_{\text {vac }} \rightarrow 0$. The zero result is in apparent contradiction with Eqs. (2.1) and (2.2). But it is not totally unexpected since it is in complete agreement with Eq. (3.4) which follows from the Gibbs-Duhem relation: in the absence of external environment the external pressure is zero, and thus the pressure of the liquid in its equilibrium ground state $p_{\mathrm{vac}}=0$. Therefore $\rho_{\mathrm{vac}}=-p_{\mathrm{vac}}=0$, and this nullification occurs irrespective of whether the liquid is made of fermionic or bosonic atoms.

If the observers living within the droplet measure the vacuum energy (or the vacuum pressure) and compare it with their estimate, Eq. (2.1) or Eq. (2.2) depending on in which liquid they live, they will be surprised by the disparity of many orders of magnitude between the estimate and observation. But we can easily explain to these observers where the mistake is. The equations (2.1) and (2.2) take into account only the degrees of freedom below the cut-off energy. If one takes into account all the degrees of freedom, not only the low-energy modes of the effective QFT, but the real microscopic energy of interacting atoms (what the low-energy observer is unable to do), the zero result will be obtained. The exact cancellation occurs without any special fine-tuning: the microscopic degrees of freedom of the system perfectly neutralize the huge contribution of the sub-Planckian modes due to the thermodynamic relation applied to the whole equilibrium ground state.

The above thermodynamic analysis does not depend on the microscopic structure of the vacuum and thus can be applied to any quantum vacuum, including the vacuum of relativistic QFT. This is another lesson from condensed matter which we may or may not accept: the energy density of the homogeneous equilibrium state of the quantum vacuum is zero in the absence of external environment. The higher-energy (trans-Planckian) degrees of freedom of the quantum vacuum perfectly cancel the huge contribution of the zero-point motion of the quantum fields to the vacuum energy. This occurs without fine-tuning because 
of the underlying general thermodynamic laws.

There exists a rather broad belief that the problem of the vacuum energy can be avoided simply by the proper choice of the ordering of the QFT operators $\psi_{a}$ and $\psi_{a}^{\dagger}$. However, this does not work in situations when the vacuum is not unique or is perturbed, which we discuss below. In our quantum liquids, the zero result has been obtained using the original pre-QFT microscopic theory - the Schrödinger quantum mechanics of interacting atoms, from which the QFT emerges as a secondary (second-quantized) theory. In this approach the problem of the ordering of the operators in the emergent QFT is resolved on the microscopic level.

\section{COINCIDENCE PROBLEM}

Let us turn to the second cosmological problem - the coincidence problem: Why is in the present Universe the energy density of the quantum vacuum of the same order of magnitude as the matter density? To answer this question let us again exploit our quantum liquids as a guide. Till now we discussed the pure vacuum state, i.e. the state without matter. In QFT of quantum liquids the matter is represented by excitations above the vacuum quasiparticles. We can introduce thermal quasiparticles by applying a non-zero temperature $T$ to the liquid droplets. The quasipartcles in both liquids are 'relativistic' and massless. The pressure of the dilute gas of quasiparticles as a function of $T$ has the same form in two superfluids if one again uses the effective metric:

$$
p_{\text {matter }}=\gamma T^{4} \sqrt{-g} .
$$

For quasipartcles in ${ }^{4} \mathrm{He}, \sqrt{-g}=c^{-3}$ is the square-root of determinant of the effective acoustic metric as before, and the parameter $\gamma=\pi^{2} / 90$; for the fermionic quasiparticles in ${ }^{3} \mathrm{He}-\mathrm{A}$, one has $\sqrt{-g}=c_{x}^{-1} c_{y}^{-1} c_{z}^{-1}$ and $\gamma=7 \pi^{2} / 360$. Such 'relativistic' gas of quasiparticles obeys the ultra-relativistic equation of state for radiation:

$$
\rho_{\text {matter }}=3 p_{\text {matter }}
$$


Let us consider again the droplet of a quantum liquid which is isolated from the environment, but now at finite $T$. The new factor which is important is the 'radiation' pressure produced by the gas of 'relativistic' quasiparticles. In the absence of environment and for a sufficiently big droplet, when we can neglect the surface tension, the total pressure in the droplet must be zero. This means that in equilibrium, the partial pressure of matter (quasiparticles) must be necessarily compensated by the negative pressure of the quantum vacuum (superfluid condensate):

$$
p_{\text {matter }}+p_{\text {vac }}=0 .
$$

The vacuum pressure leads to vacuum energy density according the equation of state (1.1) for the vacuum, and one obtains the following relation between the energy density of the vacuum and that of the ultra-relativistic matter in the thermodynamic equilibrium:

$$
\rho_{\mathrm{vac}}=-p_{\mathrm{vac}}=p_{\text {matter }}=\frac{1}{3} \rho_{\text {matter }} .
$$

This is actually what occurs in quantum liquids, but the resulting equation, $\rho_{\mathrm{vac}}=\frac{1}{3} \rho_{\mathrm{matter}}$, does not depend on the details of the system. It is completely determined by the thermodynamic laws and equation of state for matter and is equally applicable to both quantum systems: (i) superfluid condensate + quasiparticles with linear 'relativistic' spectrum; and (ii) vacuum of relativistic quantum fields + ultra-relativistic matter. That is why we can learn some more lessons from the condensed-matter examples.

Let us compare Eq. (5.4) with Eq. (1.5) which expresses the cosmological constant in terms of the matter density in the Einstein Universe also filled with the ultra-relativistic matter. The difference between them is by a factor 3. The reason is that in the effective QFT of liquids the Newtonian gravity is absent; the effective matter living in these liquids is non-gravitating: quasiparticles do not experience the attracting gravitational interaction. Our condensed matter reproduces the Universe without gravity, i.e. obeying Einstein's special theory of relativity. Thus we obtained that even without gravity, the Universe filled with hot matter can be stabilized by the vacuum, in this case the negative vacuum pressure 
counterbalances the expanding tendency of the hot gas (see also Eq. (7.1) in Sec. VII and Ref. [10]). For both worlds, with and without gravity, the Einstein prediction in Ref. [2] is correct: the matter homogeneously distributed in space induces the non-zero cosmological constant.

This and the other examples lead us to the more general conclusion: when the vacuum is disturbed, it responds to perturbation, and the vacuum energy density becomes non-zero. Applying this to the general relativity, we conclude that the homogeneous equilibrium state of the quantum vacuum without matter is not gravitating, but deviations of the quantum vacuum from such states have weight: they are gravitating. In the above quantum-liquid examples the vacuum is perturbed by the non-gravitating matter and also by the surface tension of the curved 2D surface of the droplet which adds its own partial pressure (see Sec. VII). In the Einstein Universes it is perturbed by the gravitating matter and also by the gravitational field (the 3D space curvature, see Sec. VII). In the expanding or rotating Universe the vacuum is perturbed by expansion or rotation, etc. In all these cases, the value

of the vacuum energy density is proportional to the magnitude of perturbations. Since all the perturbations of the vacuum are small in the present Universe, the present cosmological constant must be small.

The special case is when the perturbation (say, matter) occupies a finite region of the infinite Universe. In this case the pressure far outside this region is zero which gives $\lambda=0$. This is in a full agreement with the statement of Einstein in Ref. [2] that the $\lambda$-term must be added to his equations when the average density of matter in the Universe is non-zero.

\section{ENERGY OF FALSE AND TRUE VACUA}

Let us turn to some other problems related to the cosmological constant. For example, what is the energy of the false vacuum and what is the cosmological constant in such a vacuum? This is important for the phenomenon of inflation - the exponential super-luminal expansion of the Universe. In some theories, the inflation is caused by a false vacuum. It is 
usually assumed that the energy of the true vacuum is zero, and thus the energy of the false vacuum must be positive. Though the false vacuum can be locally stable at the beginning, $\lambda$ in this vacuum must be a big positive constant, which causes the exponential de-Sitter expansion. Let us look at this scenario using our knowledge of the general thermodynamic properties of the quantum vacuum.

Analyzing the Gibbs-Duhem relation we find that in our derivation of the vacuum energy, we never used the fact that our system is in the true ground state. We used only the fact that our system is in the thermodynamic equilibrium. But this is applicable to the metastable state too if we neglect the tiny transition processes between the false and true vacua, such as quantum tunneling and thermal activation. Thus we come to the following, at first glance paradoxical, conclusion: the cosmological constant in all homogeneous vacua in equilibrium is zero, irrespective of whether the vacuum is true or false. This poses constraints on some scenarios of inflation. 
FIGURES

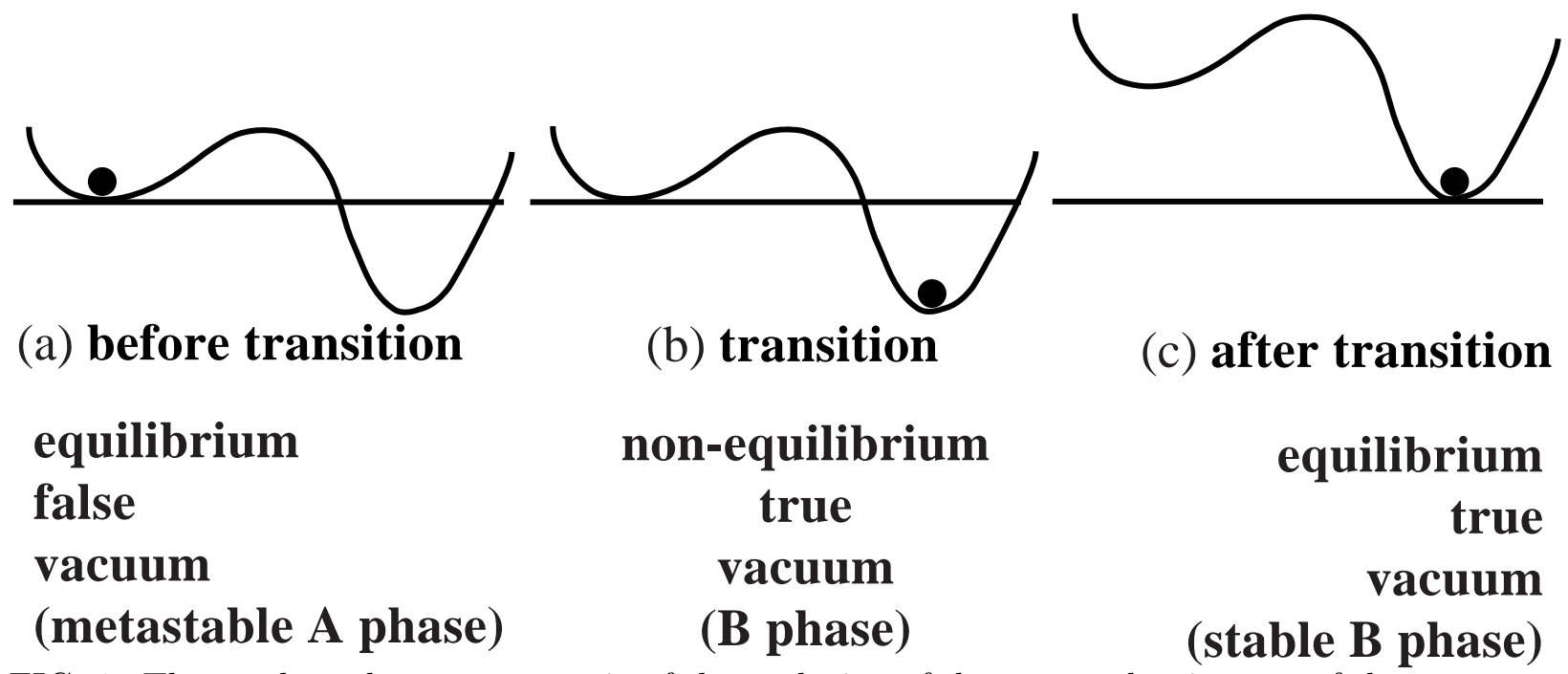

FIG. 1. The condensed-matter scenario of the evolution of the energy density $\rho_{\text {vac }}$ of the quantum vacuum in the process of the first order phase transition from the equilibrium false vacuum to the equilibrium true vacuum. Before the phase transiton, i.e. in the false but equilibrium vacuum, one has $\rho_{\text {vac }}=0$. During the transient period the microscopic parameters of the vacuum readjust themselves to new equilibrium state, where the equilibrium condition $\rho_{\mathrm{vac}}=0$ is restored.

If the vacuum energy is zero both in the false and true vacuum, then how and why does the phase transition occur? The thermodynamic analysis for quantum liquids gives us the answer to this question too. Let us consider the typical example of the first-order phase transition which occurs between the metastable quantum liquid ${ }^{3} \mathrm{He}-\mathrm{A}$ and the stable quantum liquid ${ }^{3} \mathrm{He}-\mathrm{B}$, Fig. 1. In the initial metastable but equilibrium phase $\mathrm{A}$, the thermodynamic potential for this monoatomic liquid is zero, $E_{A}-\mu_{A} N=0$. The same thermodynamic potential calculated for the phase B at the same $\mu=\mu_{A}$ is negative: $E_{B}-$ $\mu_{A} N<0$, and thus the liquid prefers the phase transition from the phase A to phase B. When the transition to the B-phase occurs, the vacuum energy becomes negative, which corresponds to the non-equilibrium state. During some transient period of relaxation towards the thermodynamic equilibrium, the parameter $\mu$ is readjusted to a new equilibrium state. After that $E_{B}-\mu_{B} N=0$, i.e. the vacuum energy density $\rho_{\mathrm{vac}}$ in the true vacuum B also becomes zero. 
We can readily apply this consideration to the quantum vacuum in our Universe. This condensed-matter example suggests that the cosmological constant is zero before the cosmological phase transition. During the non-equilibrium transient period of time, the microscopic (Planckian) parameters of our vacuum are adjusted to a new equilibrium state in a new vacuum, and after that the cosmological constant becomes zero again. Of course, we do not know what are these microscopic parameters and how they relax in the new vacuum to establish the new equilibrium. This already depends on the details of the system and cannot be extracted from the analogy with quantum vacua in liquids. However, using our experience with quantum liquids we can try to estimate the range of change of the microscopic parameters during the phase transition.

Let us consider, for example, the electroweak phase transition, assuming that it is of the first order and thus can occur at low temperature, so that we can discuss the transition in terms of the vacuum energy. In this transition, the vacuum energy density changes from zero in the initially equilibrium false vacuum to the negative value on the order of

$$
\delta \rho_{\mathrm{vac}}^{\mathrm{ew}} \sim-\sqrt{-g} E_{\mathrm{ew}}^{4}
$$

in the true vacuum, where $E_{\text {ew }}$ is the electroweak energy scale. To restore the equilibrium, this negative energy must be compensated by the adjustment of the microscopic (transPlanckian) parameters. As such a parameter we can use the value of Planck energy scale $E_{\mathrm{Pl}}$. It determines the natural scale for the vacuum energy density $\sim \sqrt{-g} E_{\mathrm{Pl}}^{4}$. This is the contribution to the vacuum energy from the modes with the Planck energy scale. When the cosmological constant is concerned, this contribution is effectively cancelled by the microscopic (transplanckian) degrees of freedom in the equilibrium vacuum, but otherwise it plays an important role in the energy balance and also in the quantum and thermodynamic fluctuations of the vacuum energy density about zero [11]. Actually the same happens with the estimate in Eq. (2.2) of the vacuum energy in quantum liquids: the Eq. (2.2) highly overestimates the magnitude of the cosmological constant, but it gives us the correct estimate of the condensation energy, which is an important part of the vacuum energy. 
Now, using the same argumentation as in quantum liquids, we can say that the variation $\delta E_{\mathrm{Pl}}$ of this microscopic parameter $E_{\mathrm{Pl}}$ leads to the following variation of the vacuum energy:

$$
\delta \rho_{\text {vac }}^{\mathrm{Pl}} \sim \sqrt{-g} E_{\mathrm{Pl}}^{3} \delta E_{\mathrm{Pl}} .
$$

In a new equilibrium vacuum, the density of the vacuum energy must be zero $\delta \rho_{\mathrm{vac}}^{\mathrm{ew}}+\delta \rho_{\mathrm{vac}}^{\mathrm{Pl}}=0$, and thus the relative change of the microscopic parameter $E_{\mathrm{Pl}}$ which compensates the change of the electroweak energy after the transition is

$$
\frac{\delta E_{\mathrm{Pl}}}{E_{\mathrm{Pl}}} \sim \frac{E_{\mathrm{ew}}^{4}}{E_{\mathrm{Pl}}^{4}} .
$$

The response of the deep vacuum appears to be extremely small: the energy at the Planck scale is so high that a tiny variation of the microscopic parameters is enough to restore the equilibrium violated by the cosmological transition. The same actually occurs at the firstorder phase transition between ${ }^{3} \mathrm{He}-\mathrm{A}$ and ${ }^{3} \mathrm{He}-\mathrm{B}$ : the change in the energy of the superfluid vacuum after transition is compensated by a tiny change of the microscopic parameter - the number density of ${ }^{3} \mathrm{He}$ atoms in the liquid: $\delta n / n \sim 10^{-6}$.

This remarkable fact may have some consequences for the dynamics of the cosmological constant after the phase transition. Probably this implies that $\lambda$ relaxes rapidly. But at the moment we have no reliable theory describing the processes of relaxation of $\lambda[12-14]$ : the dynamics of $\lambda$ violates the Bianchi identity, and this requires the modification of the Einstein equations. There are many ways of how to modify the Einstein equations, and who knows, maybe the thermodynamic principles can show us the correct one.

\section{STATIC UNIVERSES WITH AND WITHOUT GRAVITY}

As is well known there is a deep connection between Einstein's general relativity and the thermodynamic laws. It is especially spectacular in application to the physics of the quantum vacuum in the presence of an event horizon $[15,16]$ both in the fundamental and induced gravity [17]. This connection also allows us to obtain the equilibrium Einstein 
Universes from the thermodynamic principles without solving the Einstein equations. Using this derivation we can clarify how $\lambda$ responds to matter in special and general relativity.

Let us start with the Universe without gravity, i.e. in the world obeying the laws of special relativity. For the static Universe, the relation between the matter and the vacuum energy is obtained from a single condition: the pressure in the equilibrium Universe must be zero if there is no external environment, $p_{\text {total }}=p_{\text {matter }}+p_{\text {vac }}=0$. This gives

$$
\rho_{\text {vac }}=-p_{\text {vac }}=p_{\text {matter }}=w_{\text {matter }} \rho_{\text {matter }},
$$

where $p_{\text {matter }}=w_{\text {matter }} \rho_{\text {matter }}$ is the equation of state for matter. In Sec. V this result was obtained for the condensed-matter analogs of vacuum (superfluid condensate) and radiation (gas of quasiparticles with $w_{\text {matter }}=1 / 3$ ).

In the Universe obeying the laws of general relativity, the new player intervenes - the gravitational field which contributes to pressure and energy. But it also brings with it the additional condition - the gravineutrality, which states that the total energy density in equilibrium Universe (including the energy of gravitational field) must vanish, $\rho_{\text {total }}=0$. This is the analog of the electroneutrality condition, which states that both the spatially homogeneous condensed matter and Universe must be electrically neutral, otherwise due to the long-range forces the energy of the system is diverging faster than the volume. In the same way the energy density, which for the gravitational field plays the role of the density of the electric charge, must be zero in equilibrium. Actually the gravineutrality means the equation $\rho_{\text {total }}+3 p_{\text {total }}=0$, since $\rho+3 p$ serves as a source of the gravitational field in the Newtonian limit, but we have already imposed the condition on pressure: $p_{\text {total }}=0$. Thus we have two equilibrium conditions:

$$
p_{\text {total }}=p_{\text {matter }}+p_{\text {vac }}+p_{\text {gr }}=0, \rho_{\text {total }}=\rho_{\text {matter }}+\rho_{\mathrm{vac}}+\rho_{\mathrm{gr}}=0 .
$$

As follows from the Einstein action for the gravitational field, the energy density of the gravitational field stored in the spatial curvature is proportional to

$$
\rho_{\mathrm{gr}} \propto-\frac{1}{G R^{2}} .
$$


Here $G$ is the Newton constant; $R$ the radius of the closed Universe; the exact factor is $-\frac{3}{8 \pi}$, but this is not important for our consideration. The contribution of the gravitational field to pressure is obtained from the conventional thermodynamic equation $p_{\mathrm{gr}}=-d\left(\rho_{\mathrm{gr}} R^{3}\right) / d\left(R^{3}\right)$. This gives the equation of state for the energy and partial pressure induced by the gravitational field in the Universe with a constant curvature:

$$
p_{\mathrm{gr}}=-\frac{1}{3} \rho_{\mathrm{gr}} \text {. }
$$

This effect of the 3D curvature of the Universe can be compared to the effect of the 2D spatial curvature of the surface of a liquid drop. Due to the surface tension the curved boundary of the liquid gives rise to the Laplace pressure $p_{\sigma}=-2 \sigma / R$, where $\sigma$ is the surface tension. The corresponding energy density is the surface energy divided by the volume of the droplet, $\rho_{\sigma}=\sigma S / V=3 \sigma / R$. This energy density and the Laplace pressure obey the equation of state $p_{\sigma}=-(2 / 3) \rho_{\sigma}$. If there is no matter (quasiparticles), then the Laplace pressure must be compensated by the positive vacuum pressure. As a result the negative vacuum energy density arises in the quantum liquid when its vacuum is disturbed by the curvature of the boundary: $\rho_{\mathrm{vac}}=-p_{\mathrm{vac}}=p_{\sigma}=-2 \sigma / R$. This influence of the boundaries on the vacuum energy is the analog of Casimir effect [18] in quantum liquids.

Returning to the Universe with matter and gravity, we must solve the two equations (7.2) by using the equations of state for each of the three components $p_{a}=w_{a} \rho_{a}$, where $w_{\mathrm{vac}}=-1$ for the vacuum contribution; $w_{\mathrm{gr}}=-1 / 3$ for the contribution of the gravitational field; and $w_{\text {matter }}$ for matter $\left(w_{\text {matter }}=0\right.$ for the cold matter and $w_{\text {matter }}=1 / 3$ for the ultra-relativistic matter and radiation field). The simplest solution of these equations is, of course, the Universe without matter. This Universe is flat, $1 / R^{2}=0$, and the vacuum energy density in such a Universe is zero, $\lambda=0$. The vacuum is not perturbed, and thus its energy density is identically zero.

The solution of the equations (7.2) with matter gives the following value of the vacuum energy density in terms of matter density:

$$
\rho_{\mathrm{vac}}=\frac{1}{2} \rho_{\text {matter }}\left(1+3 w_{\text {matter }}\right) .
$$


It does not depend on the Newton's constant $G$, and thus in principle it must be valid in the limit $G \rightarrow 0$. However, in the world without gravity, i.e. in the world governed by Einstein's special theory of relativity where $G=0$ exactly, the vacuum response to matter in Eq. (7.1) is different. This demonstrates that the special relativity is not the limiting case of general relativity.

In the considered simple case with three ingredients (vacuum, gravitational field, and matter of one kind) the two conditions (7.2) are enough to find the equilibrium configuration. In a situation with more ingredients we can also use the thermodynamic analysis, but now in terms of the free energy which must be minimized in order to find the equilibrium Universe (see e.g. Ref. [19]).

\section{CONCLUSION}

The general thermodynamic analysis of the quantum vacuum, which is based on our knowledge of the vacua in condensed-matter systems, is consistent with Einstein's earlier view on the cosmological constant. In the equilibrium Universes the value of the cosmological constant is regulated by matter. In the empty Universe, the vacuum energy is exactly zero, $\lambda=0$. The huge contribution of the zero point motion of the quantum fields to the vacuum energy is exactly cancelled by the trans-Planckian degrees of freedom of the quantum vacuum without any fine-tuning. In the equilibrium Universes homogeneously filled with matter, the vacuum is disturbed, and the density of the vacuum energy becomes proportional to the energy density of matter, $\lambda=\rho_{\text {vac }} \sim \rho_{\text {matter }}$. This takes place even within Einstein's theory of special relativity, i.e. in a world without gravity, even though the response of the vacuum to matter without gravity is different.

So, instead of being "mein grösster Fehler", $\lambda$ appeared to be one of the brilliant inventions of Einstein. It was reinforced by the quantum field theory and passed all the tests posed by it. The thermodynamic laws hidden in Einstein's general theory of relativity proved to be more general than relativistic quantum field theory. Now we must move further - out 
of the thermodynamic equilibrium. Introducing $\lambda$, Einstein left us with the problem of how to relax $\lambda$. This is a challenge for us to find the principles which govern the dynamics of $\lambda$. What can the quantum liquids, with their quantum vacuum and effective QFT, say on that? Shall we listen to them?

I highly appreciate the hospitality extended to me during my visit to the Scuola Normale Superiore in Pisa where this paper has been written. This work was also supported in part by the Russian Foundations for Fundamental Research, by the Russian Ministry of Education and Science through the Leading Scientific School grant \#2338.2003.2 and through the Research Programme "Cosmion", and by ESF COSLAB Programme. 


\section{REFERENCES}

[1] A. Einstein, Kosmologische Betrachtungen zur allgemeinen Relativitätstheorie, Sitzungberichte der Preussischen Akademie der Wissenschaften, 1, 142-152 (1917); also in a translated version in The principle of Relativity, Dover (1952).

[2] A. Einstein, Prinzipielles zur allgemeinen Relativitätstheorie, Annalen der Physik 55, $241-244$ (1918).

[3] S. Weinberg, The cosmological constant problem, Rev. Mod. Phys. 61, 1-23 (1989).

[4] T. Padmanabhan, Cosmological constant - the weight of the vacuum, Phys. Rept. 380, $235-320(2003)$

[5] D.N. Spergel, L. Verde, H.V. Peiris, et al., First Year Wilkinson Microwave Anisotropy Probe (WMAP) Observations: Determination of Cosmological Parameters, Ap. J. Suppl. 148, 175 (2003).

[6] G.E. Volovik, The Universe in a Helium Droplet, Clarendon Press, Oxford (2003).

[7] F.R. Klinkhamer and G.E. Volovik, Emergent CPT violation from the splitting of Fermi points, hep-th/0403037; Quantum phase transition for the BEC-BCS crossover in condensed matter physics and CPT violation in elementary particle physics, JETP Lett. 80, (2004), cond-mat/0407597.

[8] W.G. Unruh, Experimental black-hole evaporation?, Phys. Rev. Lett. 46, 1351-1354 (1981).

[9] A.A. Abrikosov, L.P. Gorkov and I.E. Dzyaloshinskii, Quantum Field Theoretical Methods in Statistical Physics, Pergamon, Oxford (1965).

[10] G.E. Volovik, Vacuum energy and Universe in special relativity, JETP Lett. 77, 639-641 (2003), gr-qc/0304103.

[11] G.E. Volovik, On thermodynamic and quantum fluctuations of cosmological constant, 
Pisma ZhETF 80, 531-534 (2004), gr-qc/0406005.

[12] U. Alam, V. Sahni and A.A. Starobinsky, Is dark energy decaying? JCAP 0304002 $(2003)$.

[13] H.K. Jassal, J.S. Bagla and T. Padmanabhan, WMAP constraints on low redshift evolution of dark energy, astro-ph/0404378

[14] G.E. Volovik, Evolution of cosmological constant in effective gravity, JETP Lett. 77, 339-343 (2003), gr-qc/0302069; Phenomenology of effective gravity, in: Patterns of Symmetry Breaking, H. Arodz et al. (eds.), Kluwer Academic Publishers (2003), pp. 381-404, gr-qc/0304061.

[15] J.D. Bekenstein, Black holes and entropy, Phys. Rev. D 7, 2333 (1973).

[16] S.W. Hawking, Black hole explosions, Nature 248, 30-31 (1974).

[17] T. Jacobson, Black hole entropy and induced gravity, gr-qc/9404039.

[18] H.G.B. Casimir, On the attraction between two perfectly conducting plates ..., Kon. Ned. Akad. Wetensch. Proc. 51, 793 (1948).

[19] Carlos Barceló and G.E. Volovik, A stable static Universe? JETP Lett. 80, 239-243 (2004), gr-qc/0405105. 